\title{
The Image of Self Intersecting QCD Strings in Four Dimensions
}

\author{
Bob Bacus* and V.G.J. Rodgers ${ }^{\dagger}$ \\ Department of Physics \\ and Astronomy \\ The University of Iowa \\ Iowa City, IA 52242-1479
}

(November 5, 2018)

\begin{abstract}
We numerically examine the self-dual solutions of self-intersecting strings immersed in four dimension. By fixing a frame of reference we are able to show that self-intersecting open strings admit world sheet topologies that are able to support monopole/anti-monopole structures. Furthermore we identify the topologies that can lead to $q-\bar{q}$ production and annihilation and numerically estimate the production rate for different instantons. We find an upper bound on the production rate and use that to estimate the string tension $\mu$. By examining the pullback of $\gamma_{5}$ onto the string world sheet we show that regions of chiral symmetry breaking can exist where the string world sheet self-intersects with itself lending credence to the relationship of self-intersecting strings and instantons. We are also able to show that for torus knots, the intersection number $\nu=4(p-q)$ which validates an earlier conjecture. A program written in MAPLE is supplied to exhibit the animations of the torus knots in four dimensions. These animations are located at the website http://www-hep.physics.uiowa.edu/ ${ }^{\sim}$ bacus/research.htm under the "Animation Control Panel".
\end{abstract}

PACS: 11.25.Pm, 12.38.-t, 12.40.-y

Keywords: QCD strings, four dimensional strings, self-intersections

\section{INTRODUCTION}

Quantum Chromodynamics (QCD) is widely regarded as the correct theory of strong interactions. However, inherently non-perturbative effects such as confinement remain hidden in the theory due to the nature of the strong coupling of QCD. In 1973, t'Hooft|[1] proposed the parameter $N_{c}$ of the color gauge group be treated as a free parameter, and considered the limit $N_{c} \rightarrow \infty$ applied to the expansion of a gauge theory. The resulting expansion gives Feynman diagrams which possess the same topology as the quantized dual string model with quarks at the string ends. Also, Wilson [2] showed that lattice gauge theory in the strong-coupling approximation is a confining theory due to the formation of color charged strings formed by Faraday flux lines. However this strong coupling approximation cannot be made in the continuous theory. Later Makeenko and Migdal [| $\mid$ as well as Gervais and Nevue 3$]$ were able to establish to first order in the lattice spacing an equivalence between a Wilson loop

$$
\Phi\left(x^{\mu}(\tau)\right)=\operatorname{tr} T \exp \left(\int A_{\nu} \frac{d X^{\nu}}{d \sigma} d \sigma\right)
$$

and solutions to the single string Schroedinger equation, provided that the gauge field satisfied the Yang-Mills equations of motion. These indirect probes suggests that the effective action for QCD in the strong coupling regime is a stringlike theory. Although the precise string theory that governs strongly coupled QCD is unknown we expect this string theory to possess certain qualitative features that are characteristic of QCD. Polyakov [7] and Balachandran et al. [8] examined the effect of adding a term proportional to the extrinsic curvature to the Nambu-Goto action so that $\Theta$ vacua effects could be incorporated into the string ansatz. The extrinsic curvature term admits self-intersecting immersions as solutions and Nair and Mazur[9] relate the self-intersections number to the instanton number.

In this note we will examine these self-intersecting string immersions while in a fixed frame in four-dimensions to see if these configurations possess any realistic topological properties that we expect from an effective action of QCD. We will focus our attention on the torus knot solutions of Robertson 13 in order to be explicit. We will show that there exists topologies that supports monopole/anti-monopole fields whose flux is constrained to a finite string (tube) and in some cases to strings that are infinite in length. Furthermore we will show that there exists topologies that can support pair production and annihilation. By this we mean that the immersed world sheets have remnants that

\footnotetext{
*bacus@hepaxp.physics.uiowa.edu

†vincent-rodgers@uiowa.edu
} 
appear as finite segments of strings that emerge and dissolve in a fixed frame. These segments are related to an integer $q$ that appears in the self-dual solutions that we consider. We are able to show that $q=111$ puts an upper limit on the production of fermions. We then examine the pullback of $\gamma_{5}$ onto the world sheet and show that there can be chiral symmetry breaking "bubbles" around the points where the immersion self-intersects with itself. This helps strengthen the relationship between intersecting strings on the one hand and QCD instantons on the other. Next we verify the conjecture of Robertson [13] by showing that for torus knot solutions that the intersection number is given by $\nu=4(p-q)$. We end by providing a program in MAPLE that annimates the torus knot and torus link solutions.

\section{TOPOLOGICAL SUPPORT FOR QCD PROCESSES FROM SELF-DUAL STRINGS}

One of the questions we ask is what topologies are provided by the string to support the gauge fields and quarks in four dimensions. We are interested in the classical features of the self-intersecting instantons since these feature should appear as first order quantum corrections to the effective action of QCD in the strong coupling regime. In order to appreciate the behavior and support of the strings we need to sit in a frame and examine the topologies that the immersed string unfolds. Since the self-dual solutions have non-trivial winding number any features we find related to the self-intersection will be protected from quantum fluctuations since winding number is a topological propoerty of the string configuration. To proceed we will look at the Nambu-Goto action and include modifications introduced by Polyakov. In order to minimize the contributions of crumpled string world sheets to the effective action of QCD, Polyakov proposed the action following;

$$
S=\mu \int \sqrt{g} d \sigma d \tau+\frac{1}{\alpha_{0}} \int \sqrt{g} K_{b}^{A a} K_{A a}^{b} d \sigma d \tau
$$

where $K_{b}^{A a}$ is the extrinsic curvature tensor. However the presence of the extrinsic curvature term not only acts as a regulator [10,7] for bosonic strings but also provides an avenue for the introductions of the topological instanton solutions. Furthermore it has been shown that the presence of the extrinsic curvature is necessary for the effective action of free fermions projected onto the world sheet 11 .

The last summand to the above action can be expressed in an alternate form as;

$$
\begin{aligned}
S_{1}= & \frac{1}{\alpha_{0}} \int \sqrt{g} g^{a b} \partial_{a} t_{\mu \nu} \partial_{b} t^{\mu \nu} d \sigma d \tau \\
S_{1}= & \frac{1}{2 \alpha_{0}} \int \sqrt{g} g^{a b}\left(\left(\partial_{a} t_{\mu \nu} \mp \partial_{a} \tilde{t}^{\mu \nu}\right)\left(\partial_{b} t_{\mu \nu} \mp \partial_{b} \tilde{t}^{\mu \nu}\right) \pm\left(\partial_{a} \tilde{t}_{\mu \nu} \partial_{b} t^{\mu \nu}\right)\right) d \sigma d \tau \\
& \quad \text { where } t^{\mu \nu}=\frac{\epsilon^{a b}}{\sqrt{g}} \partial_{a} X^{\mu} \partial_{b} X^{\nu}
\end{aligned}
$$

As with QCD, the action is minimized by searching for self-dual solutions, viz. $\partial_{a} t^{\mu \nu}=\partial_{a} t^{* \mu \nu}$. Wheater 12 showed that any surface embedded in four-dimensional space-time which is a complex curve is a solution to the equations of motion for the extrinsic curvature term. Robertson 13] showed one explicit example of a self-dual string instanton is a $(\mathrm{p}, \mathrm{q})$ torus knot. These are examples of superminimal immersions into $R^{4}[14$. Pawelczyk has extended the classification of instantons and includes the Euler character of the surface as well [15]. A torus is parametrized by two variables, $u$ and $v$, representing local coordinates on the surface. The torus knot is the curve on the torus defined by the constraint $u^{p}+v^{q}=0$. Figure 1 shows an example, the $(3,2)$ torus knot. 




FIG. 1. The $(3,2)$ torus knot.

For a $(\mathrm{p}, \mathrm{q})$ torus knot instanton solution the string vector is

$$
X^{\mu}=\left[\Im\left(z^{p}\right), \Re\left(z^{p}\right), \Re\left(-z^{q}\right), \Im\left(-z^{q}\right)\right],
$$

where $z=\tau+i \sigma$ and $\Im$ and $\Re$ are the real and imaginary parts respectively. Note that Robertson has shown somewhat more generally that a string vector of the form

$$
X^{\mu}=[\Im(F(z)), \Re(F(z)), \Re(G(z)), \Im(G(z))]
$$

with $F(z)$ and $G(z)$ as any functions analytic in $z$ are also solutions to the equations of motion. These solutions automatically satisfy the Euclidean string equations

$$
\partial_{\tau} \partial_{\tau} X^{\mu}+\partial_{\sigma} \partial_{\sigma} X^{\mu}=0
$$

as well as the Euclidean constraint equations

$$
\partial_{\sigma} X^{\mu} \partial_{\tau} X^{\mu}=0
$$

and

$$
\left(\partial_{\sigma} X^{\mu}\right)^{2}=\left(\partial_{\tau} X^{\nu}\right)^{2} .
$$

To study these solutions, we create computer animated plots of the solutions over a finite time interval. We start with a particular $(\mathrm{p}, \mathrm{q})$ torus knot solution such as the $(3,2)$ knot. This is a topologically non-trivial solution with self-intersection number $\nu=4$. The solution takes the form

$$
X^{\mu}(\sigma, \tau)=\left[3 \tau^{2} \sigma-\sigma^{3}, \tau^{3}-3 \tau \sigma^{2}, \sigma^{2}-\tau^{2},-2 \tau \sigma\right]=[x, y, z, t]
$$

$X^{\mu}$ is a mapping from $(\sigma, \tau)$ space to $(x, y, z, t)$ space. We choose the last coordinate to be our time parameter and invert the the last term and replace $\tau$ in $X^{\mu}(\sigma, \tau)$ with the solution in terms of $X^{4}=t$ and $\sigma$. (One may chose other coordinates for the time such as $X^{2}$ where the $p=1$ cases correspond to the usual choice where $\tau$ is the time parameter.) In the case of the $(3,2)$ torus knot, the inversion of the last term gives $\tau=-\frac{t}{2 \sigma}$; substitution into $X^{\mu}$ gives

$$
X^{\mu}(\sigma, t)=\left[\frac{3 t^{2}}{4 \sigma}-\sigma^{3},-\frac{t^{3}}{8 \sigma^{3}}+\frac{3 t \sigma}{2}, \sigma^{2}-\frac{t^{2}}{4 \sigma^{2}}, t\right] .
$$

For each value of the time $X_{4}=t$ we generate a plot of the string. These plots are combined to form the animation. The ranges chosen were $\sigma=[-1,1], t=[-1,1]$. The open string is symmetric in our case but we are careful not to include characteristics that are due to this symmetry of the solution. We have marked the image of $\sigma=1$ and $\sigma=-1$ in order to keep track of the string endpoints on the Reimann sheet..

There are several characterisitics that we observed: 
- As opposed to finite string segments, the majority of sigments are semi-inifinte in length. This is due mainly to the inversion of the time parameter. We could have chosen $X^{2}$ to be the time component. Then for the small sector corresponding to $p=1, t$ would be $\tau$. However all other sectors would exhibit the presence of semi-infinite segments. These segments may be thought of as flux tubes that carry the monopole and anti-monopole gauge fields from a point out to infinity. One can construct gauge invariant objects that can live on these topologies (see Eq.[11],12]).

- The strings interact by exchanging flux lines. Whenever two semi-infinite segments, say A and B, touch the result is that A will pass its segment above the intersection point onto B while sewing the segment that B had above the intersection point onto its lower portion. This exchange of flux lines can also take place with segments that are finite.

- For the case when $p$ is even and $q=2$ then the image is that of a single semi-infinite segment that is actually the image of the string projected back onto itself. In this case if one had assigned a quark at $\sigma=1$ and an anti-quarks at $\sigma=-1$ then the endpoint of this type of image would correspond to a $\bar{q} q$ bound state with gauge field lines moving up and moving down the flux line that goes out to spatial infinity effectively canceling the contribution of the gauge field in the Wilson line (see Eq. 111,12]). Such configurations are expected to contribute to the $\langle\bar{q} q>$ condensate. Examples of this are the $(2,2),(4,2),(6,2)$ and $(8,2)$.

- One of the most interesting observations is the appearance and disappearance of finite segments of lines. These segments can appear at one point, interact with some segment by exchanging flux lines and then disappear at another point. The emerging segment can have a $\bar{q} q$ pair attached to the ends providing topology for pair productions and pair annihilation. We will discuss this process in some detail shortly. Nice examples are $(4,3)$, $(5,3)$ and $(1,3)$ torus knots.

- Surfaces that do not intersect $p=q$, appear as infinite or semi-infinte segments. The multiple images of the endpoints can be seen embedded in the string segment itself undergoing pair production and annihilation along the flux tube itself. This activity can also be seen in torus knots were $p$ is not relatively primed to $q$. Examples are $(6,3),(4,2)$, and $(8,4)$.

- Finally we note that the image of $\sigma= \pm 1$ does not always map into any endpoint of the image.

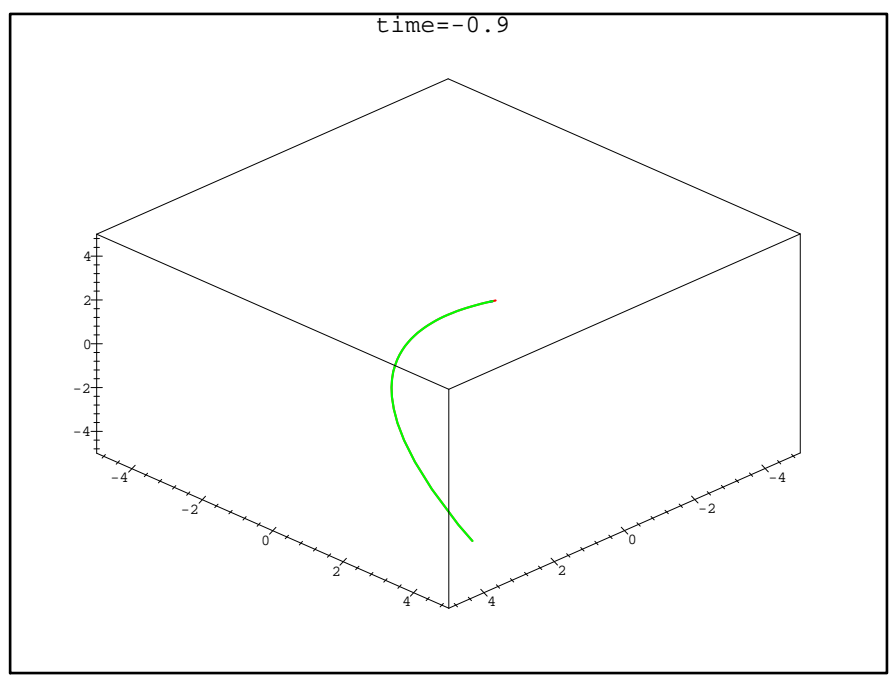

FIG. 2. The $(4,2)$ torus link instanton at time index $\mathrm{t}=-.9$.

When $p(>q)$ and $q$ and are relatively unprimed, a single semi-infinite segment appears. This is actually the image of two semi-infinite strings that mapped on top of each other. 


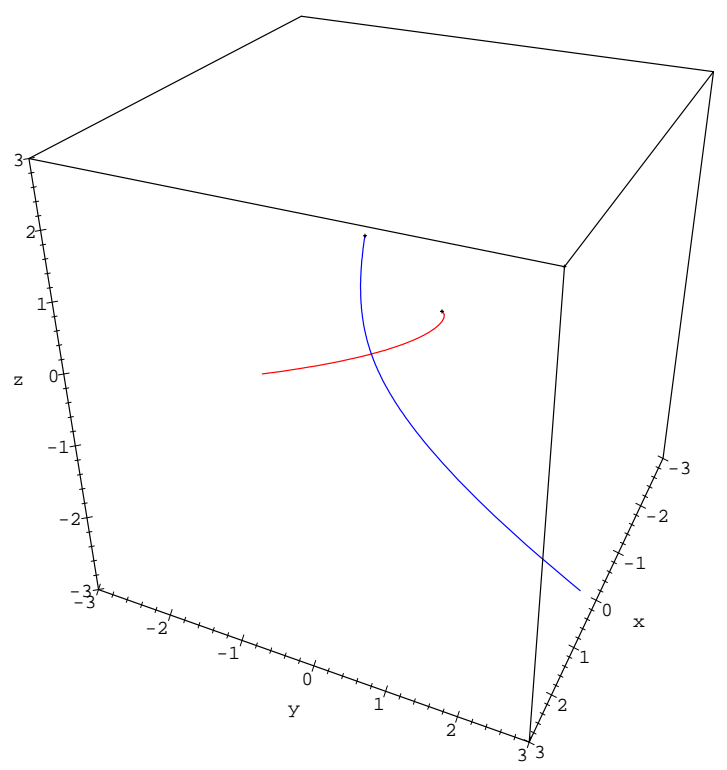

FIG. 3. The $(3,2)$ torus knot instanton at time index $\mathrm{t}=0.60$

Figure 3 shows the $(3,2)$ torus knot instanton at time $t=0.60$. The ends of the string are labeled with points, and the string is colored blue to represent $\sigma>0$ and red to represent $\sigma<0$ in order to visually distinguish these parts. The bounding box containing the plot has dimensions $x=-3 . .3, y=-3 . .3, z=-3 . .3$. In this frame we see the string broken in two halves; where part of the string around $\sigma=0$ leaves the bounding box and is mapped out to infinity.

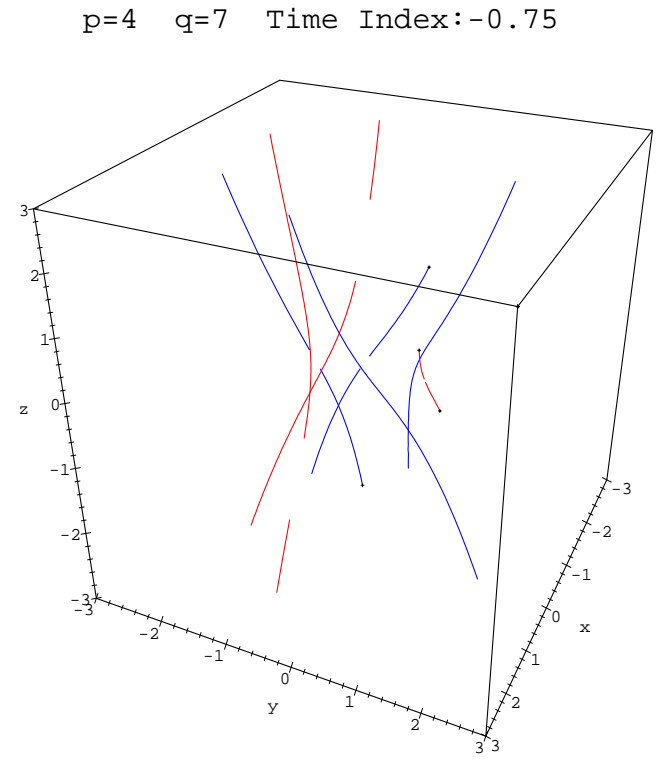

FIG. 4. The $(4,7)$ torus knot instanton at time index $\mathrm{t}=0.60$

Here is an example of a knot with negative intersection number. The vertical strands are semi-infinite in length. Here the broken strands are an imaging artifact. Just right of the center one can see the emergence of a finite segment. This segment will go on to exchange flux lines with the semi-infinite flux lines and then collapse back into the vacuum. These finite strands provide support for pair production and annihilation. 

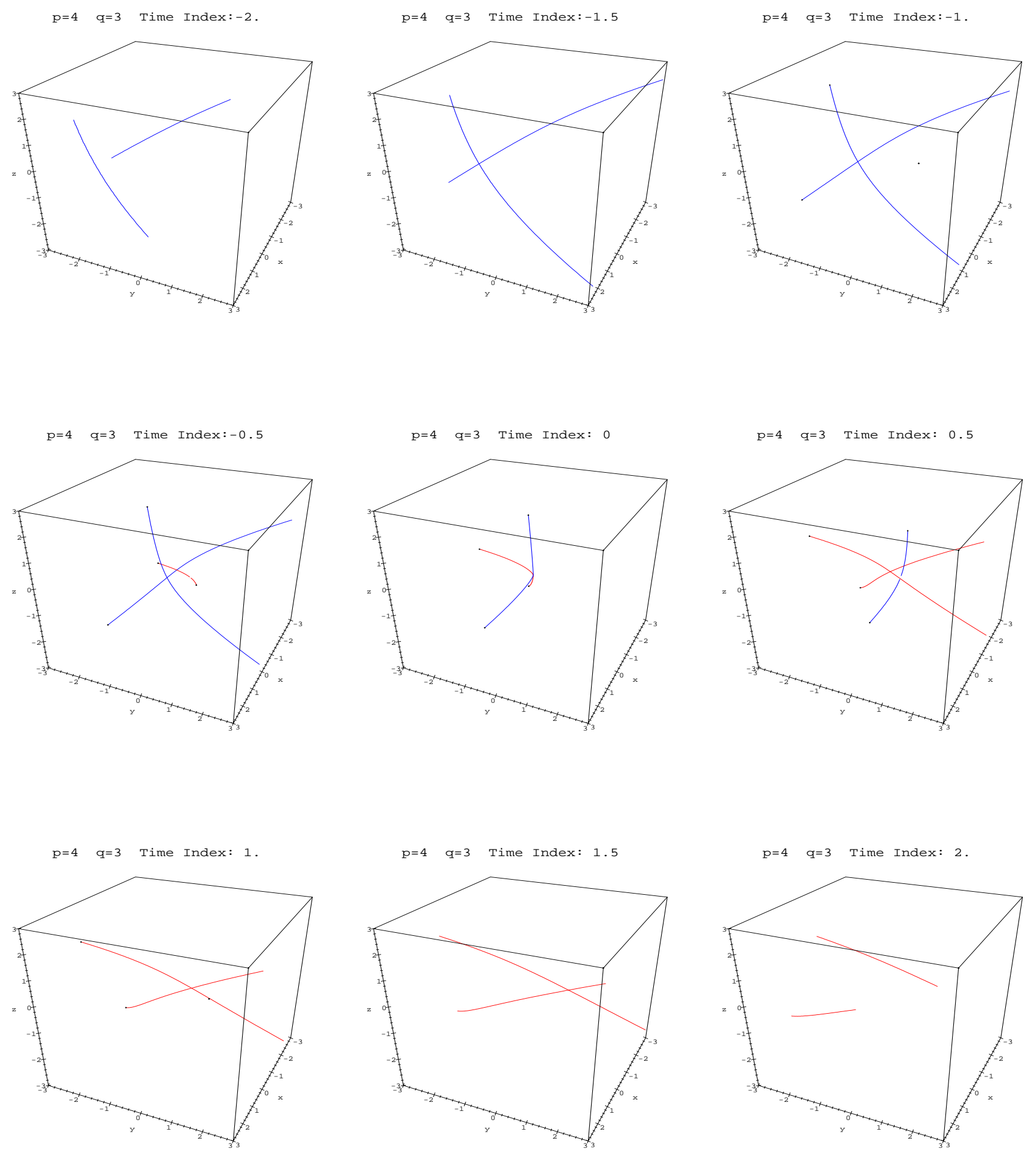

FIG. 5. Evolution of the (4,3) torus knot instanton. 

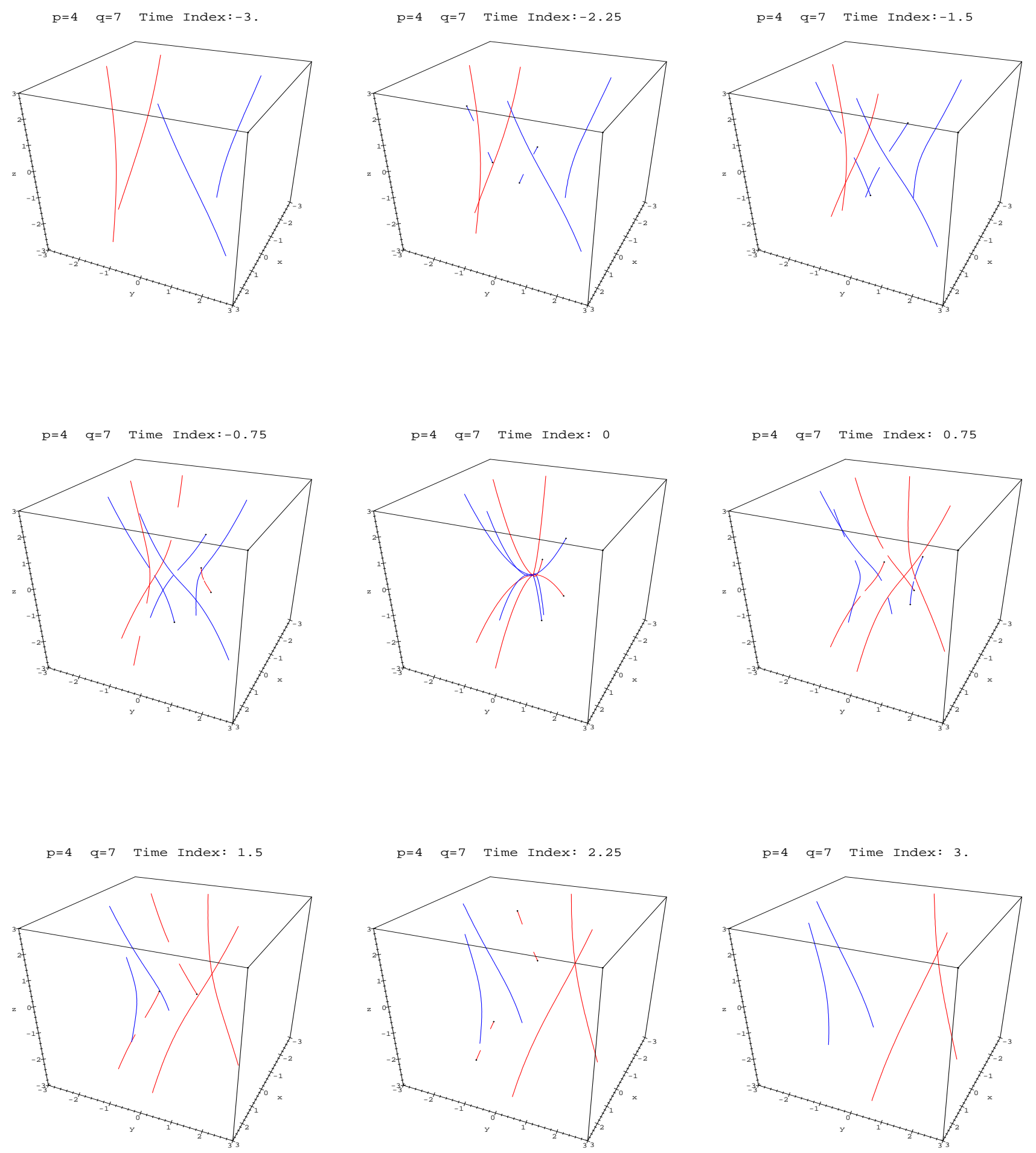

FIG. 6. Evolution of the $(4,7)$ torus knot instanton. 
Figure 5 shows a sequence of frames of the $(4,3)$ torus knot instanton. Initially, we see two string segments; at time $t \approx-1.0$, however, a pair of points appear. As time continues to evolve these points develop a string segment connecting the two. This finite segment interacts with the pair of strings initially seen, then the two original segments combine and disappear. This behavior is reminiscent of the early meson string model, in which a meson was seen as a $q \bar{q}$ pair connected by a string of gluon flux. In this interpretation, it appears as though a $q \bar{q}$ pair is created out of the vacuum, interacts strongly with a pair of gluon flux lines, then is annihilated back to the vacuum in the presence of a monopole/anti-monopole pair. Here it is the original string segment that we interpret as monopole-anti-monopole pairs. We emphasize that these are open string solutions that provide this topology as opposed to closed stings. The new requirement is that the opens strings be self intersecting.

The structure of the images suggests that the quarks interact with each other through non-local interactions. We would like for the strings to correspond to flux tubes of gauge fields and to have quarks on the endpoints. Lets fix the gauge, $A_{0}=0$ and consider the Wilson line integral given by the following path-ordered exponential,

$$
U\left(\bar{X}\left(\sigma_{+}, t\right), \bar{X}\left(\sigma_{-}, t\right)\right)=\mathcal{P} \exp \left(\int_{\sigma_{-}}^{\sigma_{+}} A_{i} \frac{d X^{i}}{d \sigma} d \sigma\right) .
$$

Here $\bar{X}\left(\sigma_{+}\right)$and $\bar{X}\left(\sigma_{-}\right)$are the end points of the string as seen from a fixed frame and $\sigma$ is used to parameterize the string for each time, $t$. From the image of the string immersions it appears that non-local point particle-like interaction Lagrangians such

$$
L=\bar{q}\left(\bar{X}\left(\sigma_{+}, t\right)\right) U\left(\bar{X}\left(\sigma_{+}, t\right), \bar{X}\left(\sigma_{-}, t\right)\right) q\left(\bar{X}\left(\sigma_{-}, t\right)\right) .
$$

can be supported. In order to account for the semi-infinite segments we can imagine taking one of the endpoints of the Wilson line out to spatial infinity. At spatial infinity the gauge fields approach zero and the Wilson line goes to the identity. The semi-infinite segment then can support a quark attached to the end of the segment with a monopole or anti-monopole gauge field confined to the segment. One can build an operator that respect the residual gauge invariance with

$$
\bar{q}\left(\bar{X}\left(\sigma_{+}, t\right)\right) U\left(\bar{X}\left(\sigma_{+}, t\right), \infty\right)
$$

or either

$$
U\left(\infty, \bar{X}\left(\sigma_{-}, t\right)\right) q\left(\bar{X}\left(\sigma_{-}, t\right)\right) .
$$

The lines that are infinite in extent correspond to both Wilson line endpoints being taken to spatial infinity. These configurations seem to be the catalyst for pair production and annihilation. There might be suppression to these infinite and semi-infinite segments if one uses the same "area law" arguments [2] one uses to suppress largely separated quark paths in pair production if the flux tubes are too far from each other. However very close pairs $\bar{q} U$ and $U q$ may be significant and could contribute to $\langle\bar{q} q\rangle$. To sum up the overall picture that these string suggests in that the general vacuum structure will consist of infinite and semi-infinite flux tubes as well as emerging and disappearing

finite tubes and stable finite strings. The gauge fields will be confined to the surfaces of these segments suggesting that monopole and anti-monopole are pervasive and where pair production seems to be catalyzed by the presence of these monopoles/anti-monopoles. The presence of the flux tubes pouring their gauge flux out at spatial infinity is already suggested by the electric Meissner effect [16. The exchange of flux tubes between pair-produced segments and the other segments suggest the charge-exchange scattering processes between fermions and charged Dirac magnetic monopoles [17.

\section{III. $\bar{Q} Q$ PRODUCTION}

The topology that supports the pair production and annihilation is not due directly to string self-intersection but is related to how the time parameter $X^{4}$ takes its values from the $\sigma-\tau$ parameter space. This is related to the fact that the solution is self-dual, however. Let us consider the multi-valued relationship of $\tau$ with $\sigma$ for a given time, $X_{4}$. Since the $X_{4}$ coordinate only depends on $q$, we can examine a time sequence of the relationship of $\sigma$ vs. $\tau$. To be explicit let us consider any solution where $q=3$. 

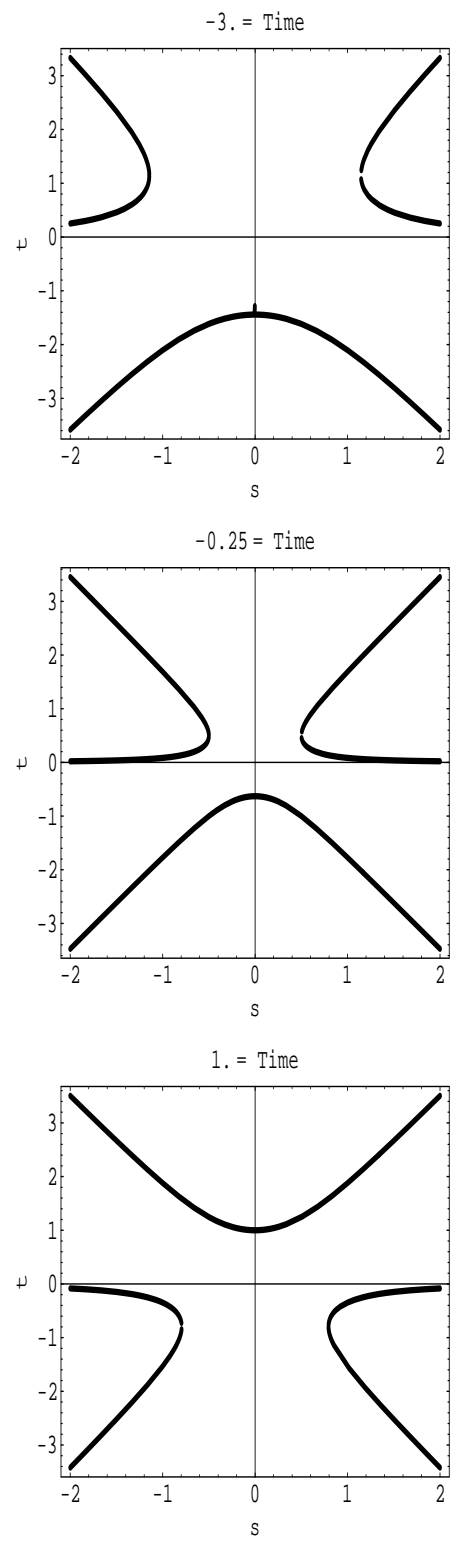
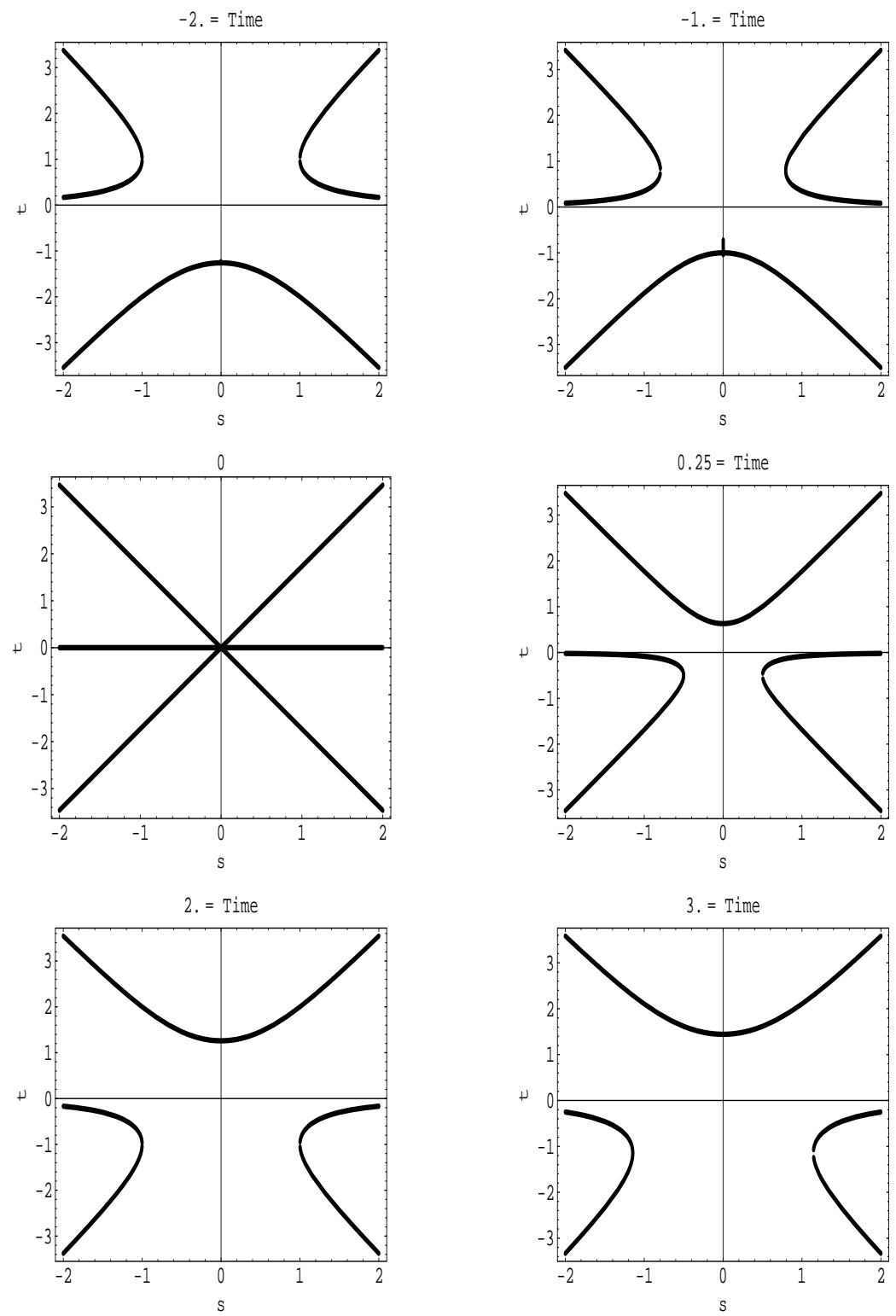

FIG. 7. $\sigma$ vs. $\tau$ at different times for $q=3$

Here one sees that at very early times $(t<<-1), \tau$ is a single valued function for values of $\sigma$ between -1 and 1. As time moves on, this function becomes multi-valued (here at $t=-2$ ) for values where $\sigma$ takes values from -1 to 1 . Now the physical $\sigma-\tau$ parameter space is split into three distinct regions corresponding to three distinct images in the frame. As time moves on these region move toward the origin until at $t=0$ all three regions merge for an "instant" and the regions begin to recede. At some later time (here $t=2$ ) the $\tau$ parameter is again single valued with respect to the physically relevant values for $\sigma$. This picture highlights two issues about the self-dual solution that may be realized in the strong coupling regime of QCD. The first is how the multivalued nature of $\tau$ vs. $\sigma$ can cause pair production and annihilation and the second being the singularity of the map at $t=0$, the point of self-intersection, leads to regions where chiral symmetry can be broken. These singular points are where the string segments are allowed to exhange flux lines and also where the determinant of the induced metric vanishes. The details of this last remark will be explored in the next section. For now we will take a closer look at the issues related to production and annihilation.

From figure [7], one sees that from the time of the production $(t=-2)$ until the time of annihilation $(t=2)$, four units of time have transpired. Here we can assume that the constant $\mu$ from the action in Eq.[1], sets the space and time scale since $\mu$ has dimensions of $\left[L^{-2}\right]$. From the uncertainty principle we can estimate a typical value of $\frac{1}{\sqrt{\mu}}$ since four quarks are produced in a time $4 \frac{1}{\sqrt{\mu}}$. This would imply that $\mu \approx\left(16 M_{q}\right)^{2}$ where $M_{q}$ is the quark mass. The $q=3$ set of torus knots correspond to the minimal pair-producing configuration. However higher $q$ instantons could easily surpass the bounds from the uncertainty principle by producing more quarks in nearly equal times to that of the $q=3$ solutions. For this reason we need to check the production capabilities of the $q>3$ solutions. 
In order to determine the time it takes for higher $q$ configurations to produce and annihilate quarks we need only to ask at what time, $t_{\text {real }}$ are all the roots of $\tau\left(\sigma=-1, t_{\text {real }}\right)$ are real. For every value of $q$ there are $q$ regions that must merge into the $(-1<\sigma<1)$ region and then move out again. The total production/annihilation time would then be $2 \times t_{\text {real }}$. As an example lets examine the $q=4$ case.
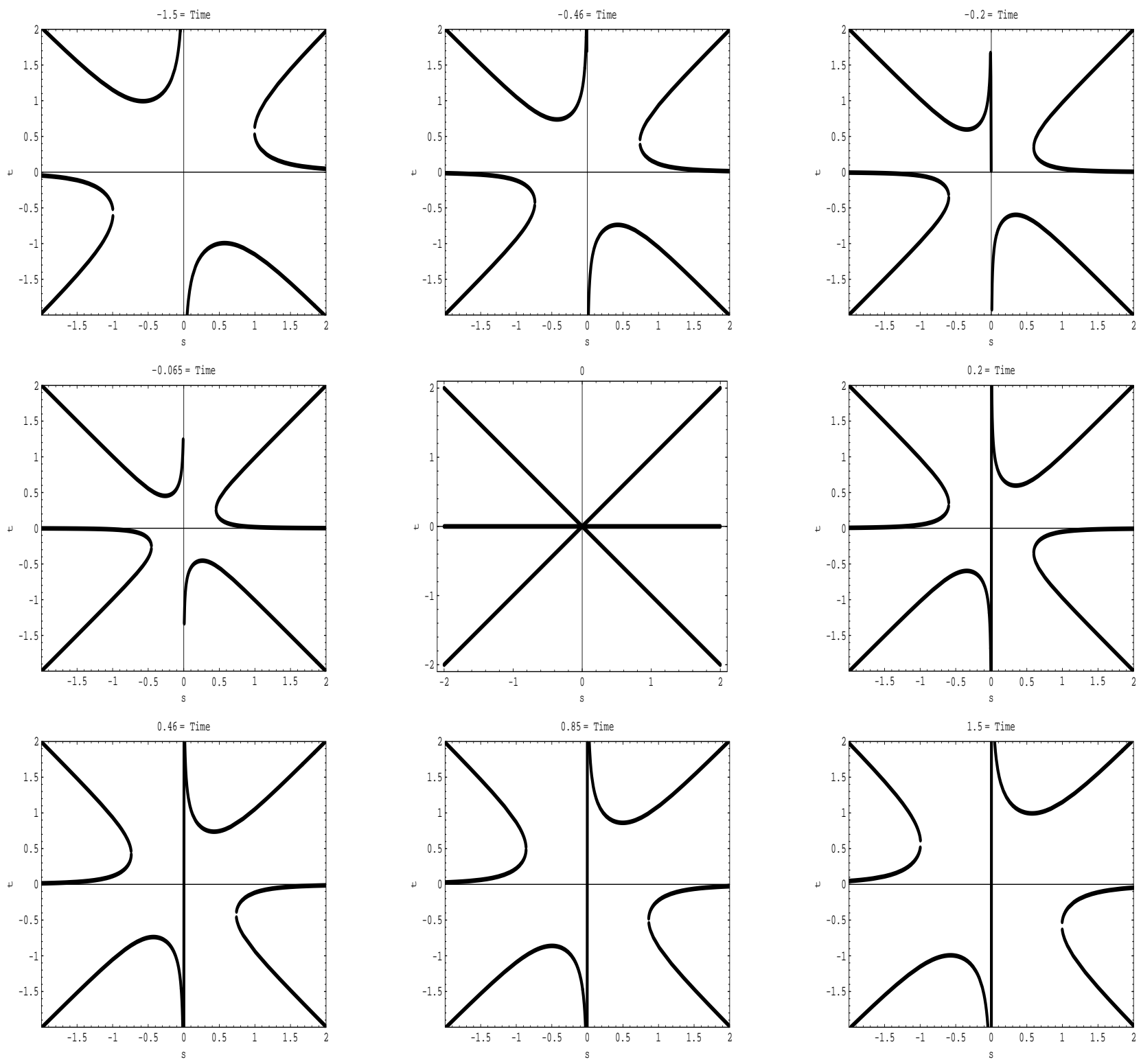

FIG. 8. $\sigma$ vs. $\tau$ at different times

In this case the pair production begins at $t=-\frac{8}{3 \sqrt{3}}$ where $\tau$ takes values of $\frac{2}{\sqrt{3}}, \frac{-1}{\sqrt{3}}$ and $\frac{-1}{\sqrt{3}}$. The time for total pair production is then $\frac{16}{3 \sqrt{3}}$ time units or about three units of time. In this amount of time again four quarks or anti-quarks are produced and annihilated. As $q$ increases it empirically appears that the amount of time to undergo full pair production monotonically decreases but reaches a plateau. One can show that the total production times for different values of $q$ are:

$$
\begin{array}{lrl}
q & =3, & t_{\text {real }}=2 \\
q & =4, & t_{\text {real }}=\frac{8}{3 \sqrt{3}} \\
q & =5, \quad t_{\text {real }}=1.37 \\
q & =6, & t_{\text {real }}=1.285
\end{array}
$$




$$
\begin{gathered}
q=10, \quad t_{\text {real }}=1.147 \\
q=100, \quad t_{\text {real }}=1.012 \\
q=111, \quad t_{\text {real }}=.608
\end{gathered}
$$

We observe that $q=111$ puts an upper limit on the value of $q$ that will produce any new pairs. For all values greater that $q=111$ some of the roots for $\tau$ remain complex even when $t=0$. By analogy with the $q=3,4$ and 5 cases we see that there can be at most 112 quarks/anti-quarks produced in 1.2 seconds by any instanton. Since the total number of quarks is limited by all torus knot instantons we can again use the uncertainty principle to get an even better estimate of $\mu$. We find that $\mu \approx\left(112 M_{q}\right)^{2}$.

Animations of several $(p, q)$ solutions with $X^{4}$ chosen as the time parameter can be found at http://wwwhep.physics.uiowa.edu/^ bacus/research.htm.

\section{CHIRAL SYMMETRY BREAKING PROCESSES}

As we discussed earlier, the self intersection of the world sheet can cause singular points to exist and lead to zeros in the determinant of the induced metric $g_{a b}=\partial_{a} X^{\nu} \partial_{b} X_{\nu}$. Self duality for the string instantons imply that $t^{\mu \nu}$ from Eq.(化) satisfy the constraint

$$
t^{\mu \nu}=\frac{1}{2} \epsilon^{\mu \nu \lambda \rho} t_{\lambda \rho}+C^{\mu \nu}
$$

where $C^{1,2}=-C^{2,1}=C^{3,4}=-C^{4,3}=1$ and all other entries are zero.

As we are interested in chiral symmetry, we would like to look at the pullback of $\gamma_{5}$ onto the string world sheet. In terms of functions on the world sheet we may write

$$
\tilde{\gamma}_{5}=\frac{\epsilon^{c d}}{4 \sqrt{g}} \hat{\epsilon}^{E F} \partial_{c} X^{\mu} \partial_{d} X^{\nu} N_{E}^{\lambda} N_{F}^{\rho} \frac{1}{4}\left[\gamma_{\mu}, \gamma_{\nu}\right]\left[\gamma_{\lambda}, \gamma_{\rho}\right]
$$

In the above $N_{A}^{\mu}$ are elements of the normal bundle of the string world sheet and $\hat{\epsilon}^{A B}$ is the volume form associated with the normal bundle. By using self duality we can eliminate the tangent vectors from the above expression and write,

$$
\tilde{\gamma}_{5}=\frac{1}{4} \hat{\epsilon}^{E F} C^{\mu \nu} N_{E}^{\lambda} N_{F}^{\rho} \epsilon_{\mu \nu \lambda \rho} \gamma_{5} .
$$

The normal vectors satisfy the conditions that $\partial_{a} X^{\mu} N_{E}^{\mu}=0$, and $N_{E}^{\mu} N^{\mu F}=\delta_{E}^{F}$. These conditions as well as the self-duality conditions imply that a solution for the tangent and normal vectors is

$$
\begin{aligned}
\partial_{\sigma} X^{\mu} & =\left(\partial_{\sigma} X^{1}, \partial_{\sigma} X^{2}, \partial_{\sigma} X^{3}, \partial_{\sigma} X^{4}\right) \\
\partial_{\tau} X^{\mu} & =\left(-\partial_{\sigma} X^{2}, \partial_{\sigma} X^{1}, \partial_{\sigma} X^{4},-\partial_{\sigma} X^{3}\right) \\
N_{1}^{\mu}= & \frac{1}{\sqrt{g}}\left(\partial_{\sigma} X^{3}, \partial_{\sigma} X^{4},-\partial_{\sigma} X^{1},-\partial_{\sigma} X^{2}\right) \\
N_{2}^{\mu}= & \frac{1}{\sqrt{g}}\left(\partial_{\sigma} X^{4},-\partial_{\sigma} X^{3}, \partial_{\sigma} X^{2},-\partial_{\sigma} X^{1}\right),
\end{aligned}
$$

where $g$ is the determinant of the induced metric. For these solutions $\sqrt{g}=g_{\sigma \sigma}$. Without loss of generality we can take the $(3,2)$ torus knot as an example. Here the torus knot determinant is

$$
\sqrt{g}=9\left(\tau^{4}+\sigma^{4}\right)-12 \tau^{2} \sigma^{2}+4 \sigma^{2}+4 \tau^{2} .
$$

Clearly this vanishes at $\sigma=0, \tau=0$. This corresponds to the point where the string self-intersects. At this point the normal bundle is ill-defined and two copies of the tangent vectors are imaged at this point. The pullback of $\gamma_{5}$ is also ill-defined when the determinant vanishes. Elsewhere on the manifold $\tilde{\gamma}_{5}$ can be used to construct a projection operator for chiral symmetry. However these points where the determinant vanish act as tiny "bubbles" where chiral symmetry is broken. The transition of the normal bundle going through this singularity has the effect of imposing reflections on some but not necessarily all of the components. In the $(3,2)$ case the relationship of the normal vectors just before and just after is given by:

$$
N_{1 \text { before }}^{\mu}=\frac{1}{\sqrt{g}}\left(\partial_{\sigma} X^{3}, \partial_{\sigma} X^{4},-\partial_{\sigma} X^{1},-\partial_{\sigma} X^{2}\right)
$$


while

$$
N_{1 \text { after }}^{\mu}=\frac{1}{\sqrt{g}}\left(\partial_{\sigma} X^{3},-\partial_{\sigma} X^{4},-\partial_{\sigma} X^{1}, \partial_{\sigma} X^{2}\right)
$$

flipping the second and fourth components. Similar changes happen in both the tangent vectors and the other normal. To show that the orientation has changed consider the vector $\bar{M}=\bar{N}_{1} \times \bar{N}_{2}$ for the $(3,2)$ knot. Just after the singularity, $M_{y} \rightarrow-M_{y}$ while all other components remain the same. This corresponds to a change in the orientation. This pictures corresponds to the field theoretic case where the core of monopoles can be thought of as bubbles where

chiral symmetry is broken. The S-wave of the fermions interact with this core which leads to processes such as those seen in 17,18$]$.

\section{THE SELF-INTERSECTION NUMBER}

It is conjectured in [13] that the self-intersection number of the $(p, q)$ torus is $\nu=4(p-q)$. Here we explicitly calculate the self-intersection number $\nu$ for a general $(\mathrm{p}, \mathrm{q})$ torus-knot solution proving the conjecture. Consider an open string where $(-L \leq \sigma \leq L)$ and $(-\infty<\tau<\infty)$. Starting from the general definition of the self-intersection number

$$
\nu=\frac{1}{2 \pi} \int_{-\infty}^{\infty} d \tau \int_{-L}^{L} d \sigma \partial_{a} t^{\mu \nu} \partial_{a} t^{\mu \nu}
$$

which we can express in terms of the string solution $X(\sigma, \tau)$ as

$$
\nu=\frac{1}{2 \pi} \int_{-\infty}^{\infty} d \tau \int_{-L}^{L} d \sigma \partial_{a}\left(\frac{\epsilon^{c d}}{\sqrt{g}} \partial_{c} X^{\mu} \partial_{d} X^{\nu}\right) \partial_{a}\left(\frac{\epsilon^{c d}}{\sqrt{g}} \partial_{c} X^{\mu} \partial_{d} X^{\nu}\right)
$$

Using the general $(\mathrm{p}, \mathrm{q})$ torus-knot solution

$$
\begin{aligned}
X(\sigma, \tau) & =\left[\left(\sigma^{2}+\tau^{2}\right)^{p / 2} \sin \left(p \tan ^{-1}\left(\frac{\sigma}{\tau}\right)\right),\left(\sigma^{2}+\tau^{2}\right)^{p / 2} \cos \left(p \tan ^{-1}\left(\frac{\sigma}{\tau}\right)\right),\right. \\
& \left.-\left(\sigma^{2}+\tau^{2}\right)^{q / 2} \cos \left(q \tan ^{-1}\left(\frac{\sigma}{\tau}\right)\right),-\left(\sigma^{2}+\tau^{2}\right)^{q / 2} \sin \left(q \tan ^{-1}\left(\frac{\sigma}{\tau}\right)\right)\right]
\end{aligned}
$$

we find

$$
\nu=\frac{1}{2 \pi} \int_{-\infty}^{\infty} d \tau \int_{-L}^{L} d \sigma \frac{8 p^{2} q^{2}(p-q)^{2}\left(\sigma^{2}+\tau^{2}\right)^{p+q-1}}{\left(p^{2}\left(\sigma^{2}+\tau^{2}\right)^{p}+q^{2}\left(\sigma^{2}+\tau^{2}\right)^{q}\right)^{2}}
$$

If we let $r^{2} \equiv \sigma^{2}+\tau^{2}$,

$$
\begin{aligned}
\nu & =\frac{1}{\pi} \int_{-\pi / 2}^{\pi / 2} d \theta \int_{0}^{\frac{L}{\cos (\theta)}} d r \frac{8 p^{2} q^{2}(p-q)^{2} r^{2(p+q-1)+1}}{\left(p^{2} r^{2 p}+q^{2} r^{2 q}\right)^{2}} \\
& =\left.\frac{4((p-q) q p)^{2}}{\pi} \int_{-\pi / 2}^{\pi / 2} d \theta \frac{1}{(p-q) q^{2}\left(q^{2} r^{2(p-q)}+p^{2}\right)}\right|^{\frac{L}{\cos (\theta)}}
\end{aligned}
$$

Let $\phi \equiv 2 \theta+\pi$,

$$
\nu=\frac{2(p-q)}{\pi} \int_{0}^{2 \pi} \frac{1}{\left(\frac{p}{q} L^{(p-q)}\right)^{2}\left(\frac{1-\cos (\phi)}{2}\right)^{(p-q)}+1} d \phi-2(p-q)
$$

Note the following relation;

$$
\int_{0}^{2 \pi} f(\sin \theta, \cos \theta) d \theta=-i \oint_{\frac{\text { unit }}{\text { circle }}} f\left(\frac{z-z^{-1}}{2 i}, \frac{z+z^{-1}}{2}\right) \frac{d z}{z}
$$

Then 


$$
\begin{aligned}
\nu & =-i \frac{2(p-q)}{\pi} \oint_{\frac{\text { unit }}{\text { circle }}} \frac{d z}{z\left(\left(\frac{p}{q} L^{(p-q)}\right)^{2}\left(\frac{1}{2}\left(1-\frac{z+z^{-1}}{2}\right)\right)^{(p-q)}+1\right)}-2(p-q) \\
& =4(q-p)-\frac{4(q-p)}{\pi} \pi \sum \text { Residues in unit circle } \\
& =4(q-p)\left(1-\sum \text { Residues in unit circle }\right)
\end{aligned}
$$

Now we must find the singular points of $f(z)=\frac{1}{z\left(\left(\frac{q}{p} L^{p-q}\right)^{2}\left(\frac{1}{2}\left(1-\frac{z+z^{-1}}{2}\right)^{p-q}+1\right)\right.}$; they are

$$
\begin{aligned}
& z=0 \\
& z=1-2\left(-\left(\frac{q}{p}\right)^{2}(L)^{2(p-q)}\right)^{\frac{1}{q-p}} \pm 2 \sqrt{\left(-\left(\frac{q}{p}\right)^{2}(L)^{2(p-q)}\right)^{\frac{1}{q-p}}+\left(\frac{q}{p}(L)^{(p-q)}\right)^{\frac{4}{q-p}}}
\end{aligned}
$$

Evaluating the residue $\left.\left(z-z_{0}\right) f(z)\right|_{z=z_{0}}$ gives zero; then we have

$$
\nu=4(q-p)
$$

for $(\mathrm{p}, \mathrm{q})$ torus-knot solutions. This confirms the conjecture of Robertson [13].

\section{COMPUTER PROGRAM}

The software described in this section was used to implement and generate the animated solutions and to perform some lengthy calculations. Two symbolic computation systems were used; Maple and Mathematica.

Listing 1 shows the Maple source code which generates raw $(x, y, z)$ coordinates for a $(\mathrm{p}, \mathrm{q})$ torus knot solution and exports them to a file. The user selects the values for $p$ and $q$, the frame numbers to start and end at, the number of data points to use for each frame, and the ranges for the parameters $t$ and $\sigma$.

\section{Listing 1}

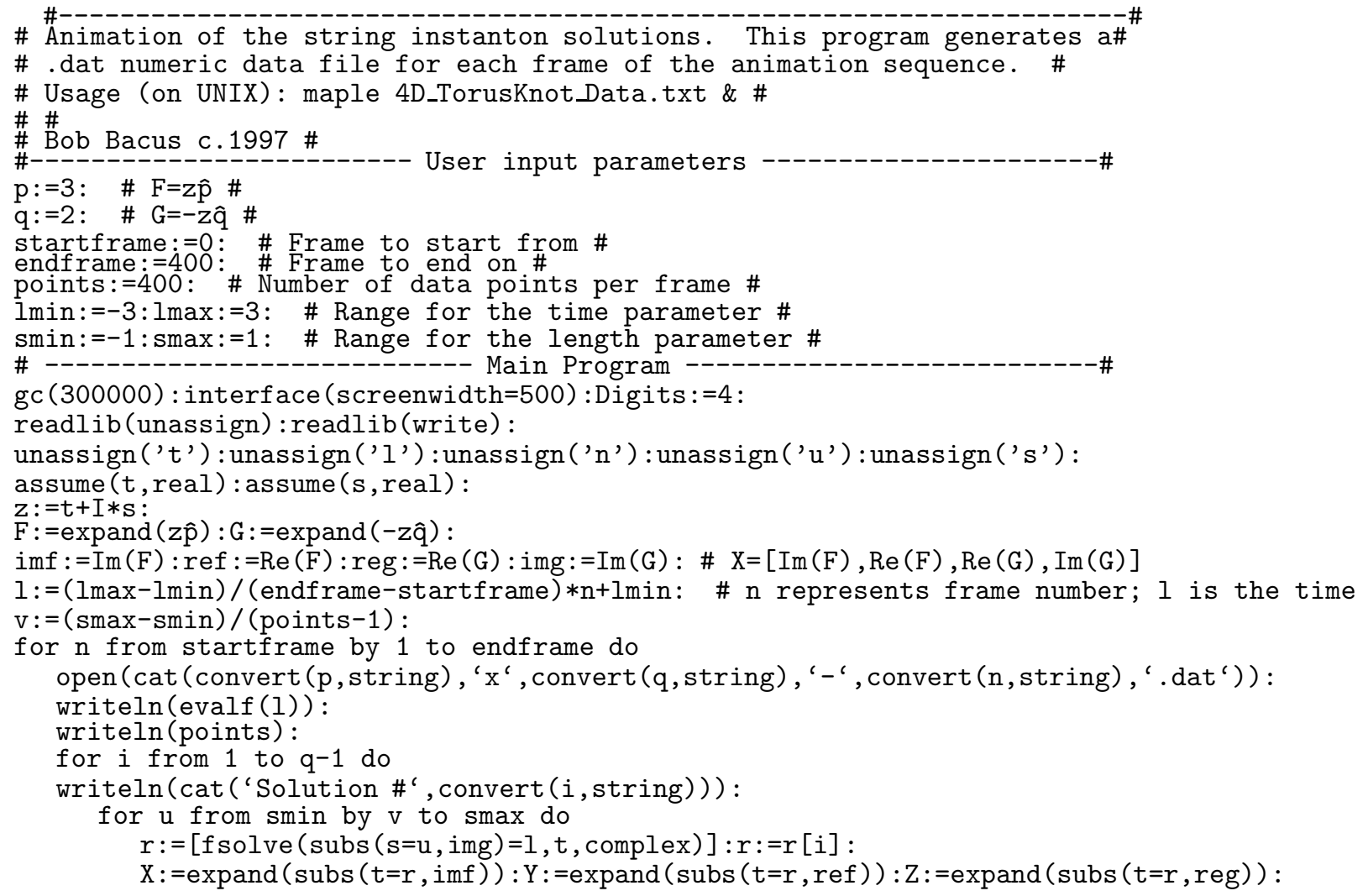




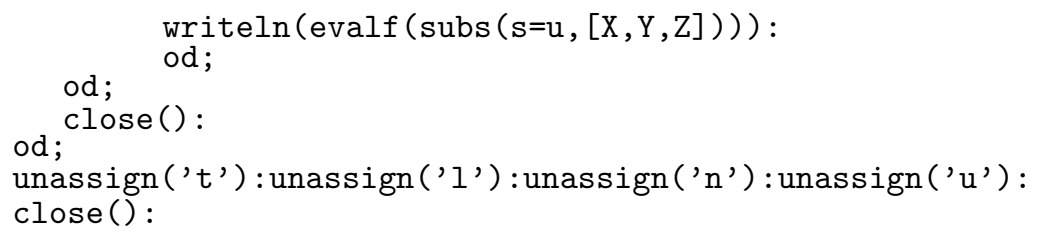

\section{Listing 2}

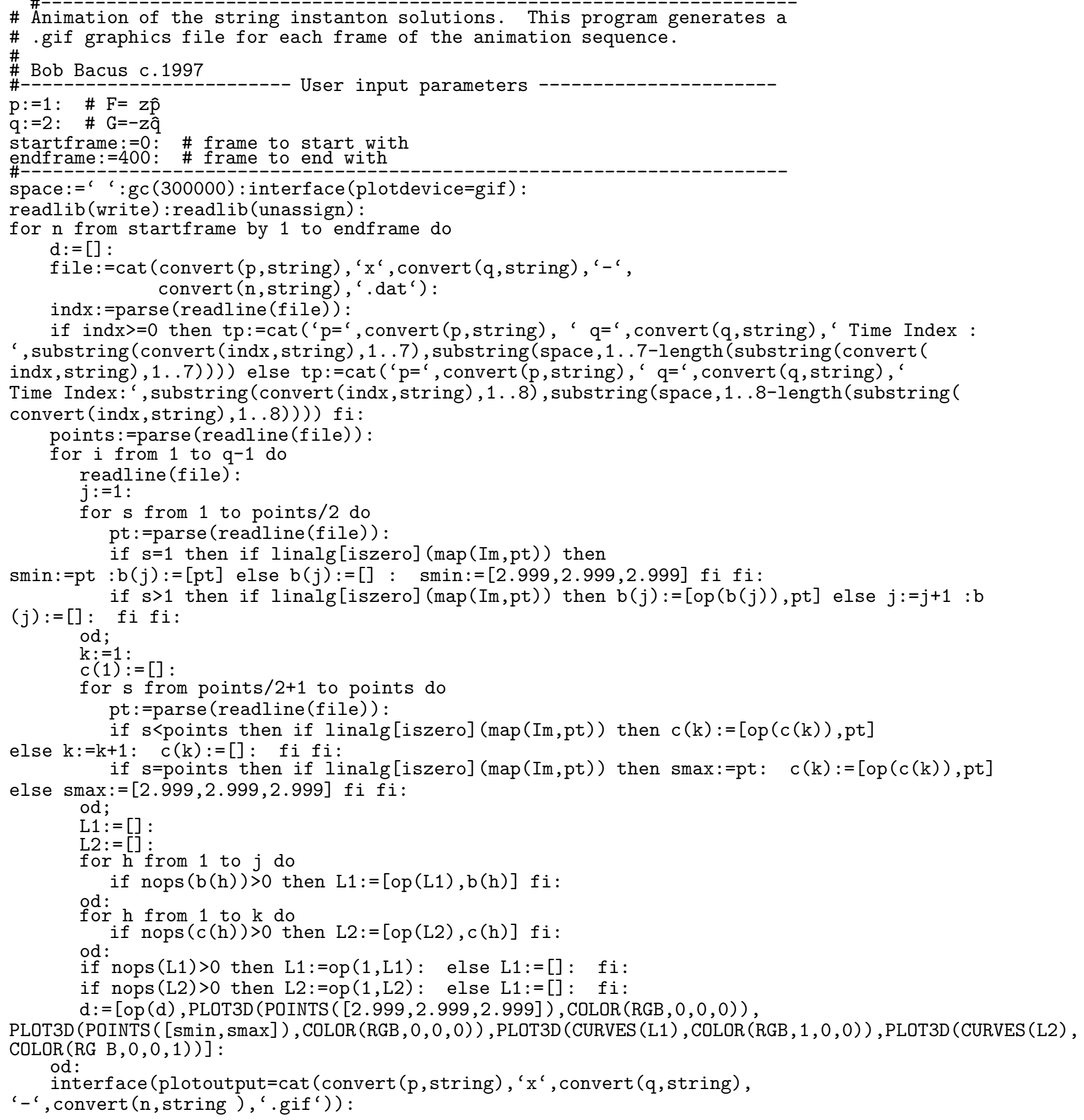


plots [display] (d, view $=[-3 . .3,-3 . .3,-3 . .3]$, projection= 1 , orientation= $[25,60]$, axes $=$ boxe

$\mathrm{d}$, labels $=[$ 'x', 'y', 'z'], scaling=constrained, titlef ont=[COURIER,10], title=tp);

test:=readline (file):

unassign('l') : unassign('b') : unassign('points') :close() :

od:

\section{ACKNOWLEDGEMENTS}

V.G.J. Rodgers thanks V.P. Nair for discussion.

\section{REFERENCES}

1. G. t'Hooft, Nucl. Phys. B72 (1973) 461

2. K. G. Wilson, Phys. Rev. D 10 (1974) 2445

3. J.-L. Gervais and A. Neveu, Phys. Lett. B80 (1979) 255

4. Yu M. Makeenko and A.A. Migdal, Phys. Lett. B88 (1979) 135

5. A. A. Belavin, A. M. Polyakov, A. Schwartz, Y. Tyupkin, Phys. Lett. 59B (1975) 85

6. E. Witten, Phys. Rev. Lett. 38 (1977) 121

7. A. M. Polyakov, Nucl. Phys. B268 (1986) 406

8. A.P. Balachandran, F. Lizzi, G. Sparano, Nucl. Phys. B263 (1986) 608

9. P.O. Mazur and V.P. Nair, Nucl. Phys. B284 (1986) 146

10. J. Ambjorn and B. Durhuus, Phys. Lett. B188 (1987) 253

11. R.Parthasarathy and K.S.Viswanathan, Lett.Math.Phys. 48 (1999) 243

12. J. F. Wheater, Phys. Lett. B208 (1988) 388

13. G. Robertson, Phys. Lett. B 226 (1989) 244

14. B.G. Konopelchenko and G. Landolfi, Phys.Lett. B459 (1999) 522

15. J.Pawelcyzk, Phy. Rev. Lett 74 (1995) 3924; Phys. Lett. B387 (1996) 287; Nucl. Phys. B491, (1997) 515

16. V.P. Nair and C. Rosenzweig, Phys. Lett. B135 (1984) 450; Phy. Rev. D31 (1985) 401

17. Allan S. Blaer, Norman H. Christ, and Ju-Fei Tang, Phy. Rev. D25 (1982) 25

18. C.G. Callan, Jr. Phys. Rev. D25 (1982) 2141 